\title{
The Multiply Imaged Strongly Lensed Supernova Refsdal
}

\author{
Patrick Kelly \\ Astronomy Department, University of California, Berkeley, \\ 501 Campbell Hall \#3411 \\ Berkeley, CA 94720 \\ email: pkelly@astro.berkeley.edu
}

\begin{abstract}
In 1964, Sjur Refsdal first considered the possibility that the light from a background supernova could traverse multiple paths around a strong gravitational lens towards us. He showed that the arrival times of the supernova's light would depend on the cosmic expansion rate, as well as the distribution of matter in the lens. I discussed the discovery of the first such multiply imaged supernova, which exploded behind the MACS J1149.6+2223 galaxy cluster. We have obtained Hubble Space Telescope grism and ground-based spectra of the four images of the supernova, which form an Einstein Cross configuration around an elliptical cluster member. These spectra as well as rest-frame optical light curves have allowed us to learn about the properties of the peculiar core-collapse supernova explosion, which occurred $4.3 \mathrm{Gyr}$ after the Bang Bang, and contain information about the lenses matter distribution as well as their stellar populations. A delayed image of the supernova is expected close to the galaxy cluster center as early as this Fall, and will serve as an unprecedented probe of the potential of a massive galaxy cluster.
\end{abstract}

Keywords. (stars:) supernovae: individual (SN Refsdal), gravitational lensing, galaxies: clusters: individual (MACS J1149.6+2223)

\section{Introduction}

Supernova (SN) surveys have sought, for many decades, to find a multiply imaged gravitationally lensed explosion. Refsdal (1964) first considered the possibility that a galaxy lens could form multiple images of a well-align background SN explosion. He showed that, for a simple lens, the relative time delays between the arrivals of the SN light in the multiple images could be used to constrain the cosmic expansion rate.

As light from a background source travels towards us through a gravitational lens, the passage of the photons is slowed due to the local curvature of space and gravitational time dilation. If the gravitational lens has a simple potential, this 'Shapiro delay' can be estimated precisely. The remaining time delay is geometric. Since the angles between the images are readily measured, the time delays, after removing the Shapiro delay, make it possible to determine an absolute distance scale, and consequently the rate of cosmic expansion.

In 2010, the Pan-STARRS collaboration discovered an unusually bright H-poor SN, PS1-10afx, with redshift $z=1.38$, and suggested that the explosion was a new species of super-luminous SN (Chornock et al. 2013). Deep subsequent optical spectroscopy along the line of sight to the SN, however, showed evidence for a foreground galaxy lens, and provided evidence that the event was instead a SN Ia magnified by a factor of $\sim 30$ (Quimby et al. 2013, 2014). In that case, available ground-based data could not resolve the multiple images of the SN which would have been separated by $<1^{\prime \prime}$. 


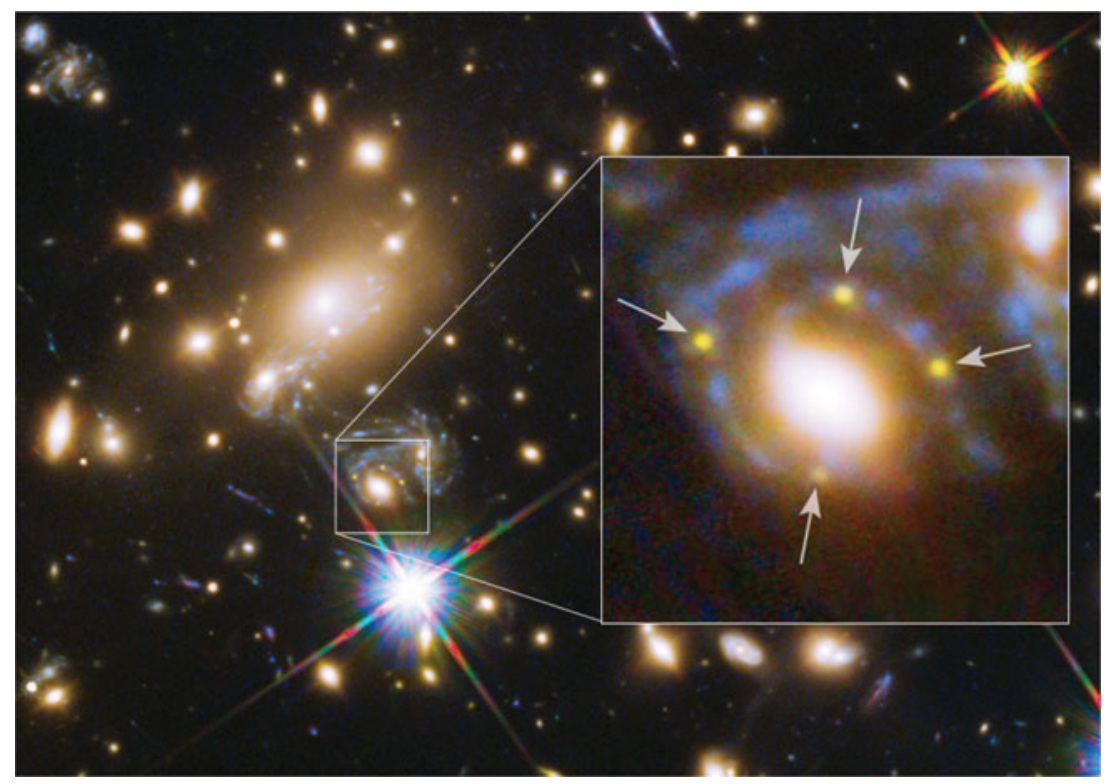

Figure 1. The first-ever strongly lensed, multiply imaged SN Refsdal. The SN is strongly lensed by both an early type cluster member and the MACS J1149 cluster, and will reappear closer to the cluster center.

In Kelly et al. (2015), we reported the discovery of the first multiply imaged, strongly lensed SN in the MACS J1149.6+2223 (Ebeling et al. 2001) galaxy-cluster field. The SN was detected in near-infrared Hubble Space Telescope (HST) WFC3 imaging taken on 11 November 2014 to calibrate grism spectroscopy acquired as part of the Grism LensAmplified Survey from Space (GLASS; GO-13459; PI: Treu; Schmidt et al. 2014; Treu et al. 2015). In late December 2014, we obtained thirty orbits of WFC3 near-infrared grism spectra of the SN and galaxy-cluster field (PI: Kelly; GO/DD-14041) to identify the spectroscopic type of the SN and measure the properties of the explosion. We have also been able to construct excellent near-infrared (rest-frame optical) light curves of the four images of SN Refsdal using data taken as part of the Frontier Fields program (PI: Lotz; GO-13504), the FrontierSN program (PI: Rodney; GO-13790), and during the grism follow-up observations.

Models of the galaxy-cluster potential predict that the SN will reappear in a different image of the redshift $z=1.49$ host galaxy that is $\sim 8^{\prime \prime}$ closer the center of the galaxy cluster field as early as Fall 2015 (Kelly et al. 2015; Oguri et al. 2015; Diego et al. 2015). This expected appearance has the greatest time delay, since its photons traverse the steepest region of the cluster potential.

We are measuring the relative time delays and magnifications of the four images discovered in November 2014 from their high-cadence light curves. The grism spectroscopy, as well as a ground-based measurement taken near maximum light in May and June 2015, have revealed key information about the SN explosion and its massive progenitor. Finally, a Cycle 23 HST program (PI: Kelly; GO-14199) will search for the expected reappearance of the SN, and allow a precise measurement of its time delay. While current constraints on the complex galaxy-cluster potential will preclude a competitive measurement of $H_{0}$ from the time delay, it will be possible to perform a precise, unprecedented measurement of a difference in the gravitational potential of the galaxy cluster close to its center. 
The light curves will also enable us to search for microlensing fluctuations that may provide useful constraints on the stellar populations both in the early type galaxy lens and within the intracluster light (Dobler \& Keeton 2006). Studying these small fluctuations requires careful processing of the imaging data, and subtraction of the diffraction spikes from a nearby bright star.

\section{References}

Chornock, R., et al. 2013, ApJ, 767, 162

Diego, J. M., et al. 2015, ArXiv: 1504.05953

Dobler, G. \& Keeton, C. 2006, ApJ, 653, 1391

Ebeling, H., et al. 2001, ApJ, 553, 668

Oguri, M. 2015, MNRAS, 449, L86

Kelly, P., et al. 2015, Science, 347, 1123

Schmidt, K. B., et al. 2014, ApJL, 782, L36

Sharon, K. \& Johnson, T. L. 2015, ApJL, 800, L26

Treu, T., et al. 2015, ApJ, 812, 114

Quimby, R. M., et al. 2013, ApJ, 768, L20

Quimby, R. M., et al. 2014, Science, 344, 396-399 